\title{
ROBUST CONTROL FOR COAXIAL TWO-WHEELED ELECTRIC VEHICLE
}

Jia-Sheng Hu

Department of Greenergy, National University of Tainan, Tainan, Taiwan,R.O.C. Institute of Industrial Science, University of Tokyo, Tokyo, Japan, jogson@ieee.org

Mi-Ching Tsai

Department of Mechanical Engineering, National Cheng Kung University, Tainan, Taiwan, R.O.C

Feng-Rung $\mathrm{Hu}$

Department of Mathematical Education, National Taichung University, Taichung, Taiwan, R.O.C.

Yoichi Hori

Institute of Industrial Science, University of Tokyo, Tokyo, Japan.

Follow this and additional works at: https://jmstt.ntou.edu.tw/journal

Part of the Engineering Commons

\section{Recommended Citation}

Hu, Jia-Sheng; Tsai, Mi-Ching; Hu, Feng-Rung; and Hori, Yoichi (2010) "ROBUST CONTROL FOR COAXIAL TWOWHEELED ELECTRIC VEHICLE," Journal of Marine Science and Technology. Vol. 18: Iss. 2, Article 3.

DOI: $10.51400 / 2709-6998.2316$

Available at: https://jmstt.ntou.edu.tw/journal/vol18/iss2/3

This Research Article is brought to you for free and open access by Journal of Marine Science and Technology. It has been accepted for inclusion in Journal of Marine Science and Technology by an authorized editor of Journal of Marine Science and Technology. 


\section{ROBUST CONTROL FOR COAXIAL TWO-WHEELED ELECTRIC VEHICLE}

Acknowledgements

This work was supported by the National Science Council (NSC) of Taiwan, R.O.C., under grant project NSC 93-2213- E-006-008. The authors would like to thank Mr. Michael J. Newkirk and anonymous reviewers for helping to improve the quality of this paper. 


\title{
ROBUST CONTROL FOR COAXIAL TWO-WHEELED ELECTRIC VEHICLE
}

\author{
Jia-Sheng $\mathrm{Hu}^{*}, * * * *$, Mi-Ching Tsai**, Feng-Rung $\mathrm{Hu}^{* * *}$, and Yoichi Hori****
}

Key words: coaxial two-wheeled electric vehicle, pilot control, robust control.

\begin{abstract}
This paper investigates robust motion pilot control of a coaxial two-wheeled electric vehicle (CTWEV) where the feedback control system is solely based on the angle of inclination without using any velocity information. $H_{\infty}$ control is employed to design a robust stabilizing controller. The proposed scheme involves an electronic differential steering algorithm to pilot the CTWEV via a joystick. Finally, evaluation examples and simulations are provided to demonstrate the effectiveness of the system.
\end{abstract}

\section{INTRODUCTION}

Research on auto-balancing robots has become a popular topic in robotics and mechatronics fields, especially twowheeled mobile robots. Due to a mobility that is superior to that of three or four-wheeled robots, the importance of auto-balancing two-wheeled robots in autonomous robot research is on the rise. The two-wheeled vehicles can also be divided into coaxial and non-coaxial types. In a non-coaxial two-wheeled vehicle, the two wheels are not devised on the same axis. In fact, this type of two-wheeled vehicle is a very common sight, such as scooters and bicycles. To operate this type of vehicle, the user should coordinate the horizontal balancing. Conversely, the coaxial two-wheeled vehicle is comprised of wheels devised on the same axis, which has become an innovative transportation vehicle in recent years. The coaxial two-wheeled electric vehicle (CTWEV) is belonging to the coaxial one. Figure 1 shows the physical wheels configuration of the CTWEV. According to the Japan Times

Paper submitted 09/12/08; revised 04/06/09; accepted 04/13/09. Author for correspondence: Jia-Sheng Hu (e-mail: jogson@ieee.org).

*Department of Greenergy, National University of Tainan, Tainan, Taiwan, R.O.C.

**Department of Mechanical Engineering, National Cheng Kung University, Tainan, Taiwan, R.O.C.

***Department of Mathematical Education, National Taichung University, Taichung, Taiwan, R.O.C.

****Institute of Industrial Science, University of Tokyo, Tokyo, Japan.
[20], the first auto-balancing two-wheeled robot was proposed by Yamafuji et al. in 1986. Their research, such as presented in [5], catalyzed much of today's inspiration as seen in subsequent studies. Currently, the "HT" and "iBOT", created by Segway [9] and Independence Technology [10], respectively, are commercially available products that have attracted many remarkable investigations into the coaxial two-wheeled electric vehicles. The CTWEV can be used in many applications, such as transportation, entertainment, security, and military affairs. The CTWEV is a naturally unstable system that looks like an inverted mobile pendulum, and its dynamics are much more complicated than its conventional counterparts. In recent years, the development of intelligent autonomous robots and remote-controlled wheeled robots are noteworthy [14]. In this analysis, joystick manipulation of the CTWEV will also be examined.

The CTWEV is of a type of mobile robot, whose motion and balancing management can be implemented using many approaches, such as linear state feedback, feedback linearization, fuzzy logic, bio-inspired suppression control, and Lyapunov control $[6,7,11,13,16,17,18,19,22,24]$. Studies [2] and [8] focus on the cost-effective CTWEV system and also show the viability of their designs. It should be noted, that most approaches originate from the Full Information (FI) [4] control setup. Therefore, in these studies, gyros are important because they are the feedback sensors that perform control operations. Although the FI approach can directly stabilize the modes of a plant using feedback control, it requires sensors for full state information. Because the CTWEV is a multi-input and multi-output (MIMO) large-scale system, the use of full state feedback control to construct this system inevitably requires many sensors. For example, Segway's "HT" system requires five gyros (velocity sensors) and two inclinometers (position sensors). Please note that the acceleration information is unnecessary in the FI approach. However, in some intelligent control approaches, such as [13] and [16], the acceleration information that assists stabilization performance of the CTWEV is required. Due to the often expensive cost of sensors, the control systems based on these techniques are not always practical solutions when considering the cost. Although these methods are all reliable CTWEV solutions, cost issues could affect its controller design. Undoubtedly, if the CTWEV is considered for some specialized tasks such as transportation, these approaches call for more extensive finances to fabricate such a vehicle. 


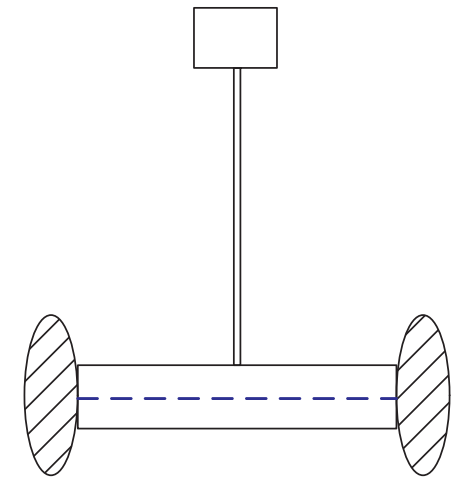

Fig. 1. Physical wheels configuration of the CTWEV.

It is known in [22] that to stabilize the CTWEV, only the cart's angle of inclination is needed. However no practical approach has been proposed to prove its feasibility. This conclusion inspires our interests to design a robust CTWEV solely based on the cart's inclined displacement. Therefore, we propose a new CTWEV remote pilot control scheme solely based on the cart's angle of inclination feedback information. Our pilot is devoted to develop a remote-controllable CTWEV for transportation use. This research is structured as follows. Section II describes the modeling issue of the CTWEV. The details about the controller design and electronic differential strategies are revealed in Section III. Section IV gives some numerical examples for evaluation. Finally, Section V offers some concluding remarks.

\section{CTWEV MODEL}

After the study done by Yamafuji et al., an increasing number of innovative auto-balancing two-wheeled robots and vehicles have been developed, such as "Yamabico Kurara" [7], "JOE" [6] and "B2" [11]. Figure 2 shows a prototype of the CTWEV studied herein where the gear box decelerates 3 times that of the motor shaft speed. The CTWEV, with a height of $0.65 \mathrm{~m}$ and a weight of $30 \mathrm{Kg}$, is driven by two 300 $\mathrm{W}$ blushless DC motors. Table 1 gives the notation definitions in SI units of the presented CTWEV system. Some discussions about CTWEV modeling can be found in $[6,7,11,17$, 18]. The readers can find the analysis in our previous study [22] for the CTWEV modeling. Here from [22], we have the CTWEV nonlinear model as

$$
\ddot{\theta}=\frac{\frac{m l \cos \theta\left(\frac{T_{L}}{R}+\frac{T_{R}}{R}+m l \dot{\theta}^{2} \sin \theta\right)}{M+2 M_{w}+m+\frac{2 J_{w}}{R^{2}}}-m g l \sin \theta}{\frac{m^{2} l^{2} \cos ^{2} \theta}{M+2 M_{w}+m+\frac{2 J_{w}}{R^{2}}}-\left(m l^{2}+J_{m \theta}+J_{p \theta}\right)},
$$

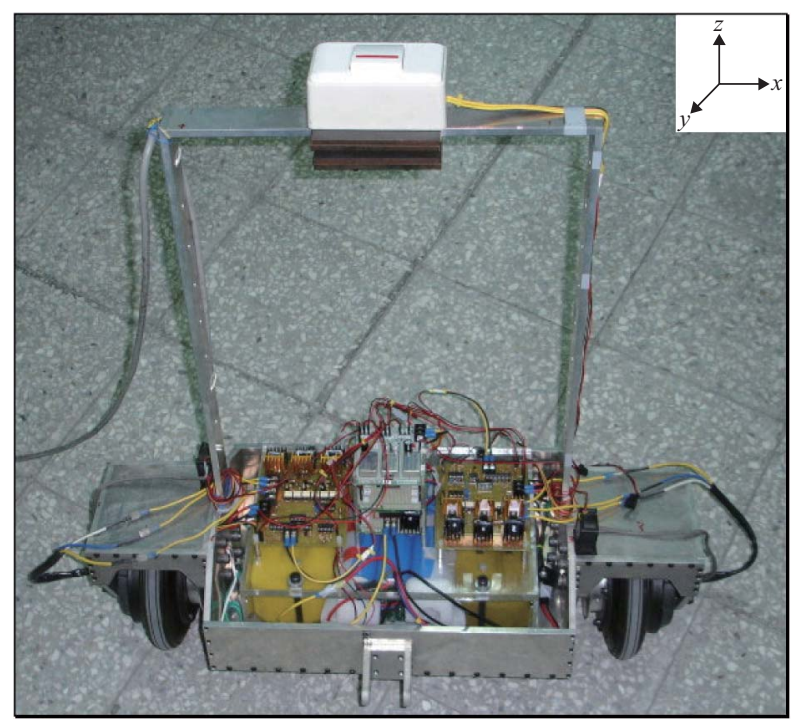

Fig. 2. A prototype of the CTWEV.

Table 1. CTWEV notations.

\begin{tabular}{|c|c|}
\hline$J_{\Theta}$ & $\mathrm{Z}$ axis, whole cart moment of inertia \\
\hline$J_{p \theta}$ & $\mathrm{X}$ axis, pendulum moment of inertia \\
\hline$J_{m \theta}$ & $\mathrm{X}$ axis, mainframe moment of inertia \\
\hline$J_{w}$ & Single wheel moment of inertia \\
\hline$M$ & Mainframe mass \\
\hline$m$ & Pendulum payload mass \\
\hline$M_{w}$ & Single wheel mass \\
\hline$H_{f}$ & Length between two wheels \\
\hline$l$ & Pendulum length \\
\hline$\theta$ & Pendulum tilt angle \\
\hline$R$ & Wheel radius \\
\hline$\Theta$ & Forward path turning angle \\
\hline$x_{M}$ & Center cart trajectory \\
\hline$T_{L}$ & Left motor driving torque \\
\hline$T_{R}$ & Right motor driving torque \\
\hline
\end{tabular}

$$
\begin{gathered}
\ddot{x}_{M}=\frac{\frac{T_{L}}{R}+\frac{T_{R}}{R}+m l \dot{\theta}^{2} \sin \theta-\frac{m^{2} l^{2} g \sin \theta \cos \theta}{m l^{2}+J_{m \theta}+J_{p \theta}}}{M+2 M_{w}+m+\frac{2 J_{w}}{R^{2}}-\frac{m^{2} l^{2} \cos \theta^{2}}{m l^{2}+J_{m \theta}+J_{p \theta}}}, \\
\ddot{\Theta}=\frac{H_{f}}{J_{\Theta}+\frac{\left(J_{w}+R^{2} M_{w}\right) H_{f}^{2}}{2 R^{2}}}\left(\frac{T_{L}}{R}-\frac{T_{R}}{R}\right) .
\end{gathered}
$$

Then a linearized model of the nonlinear plant at the equilibrium point $\theta=0^{\circ}$ can be found as 


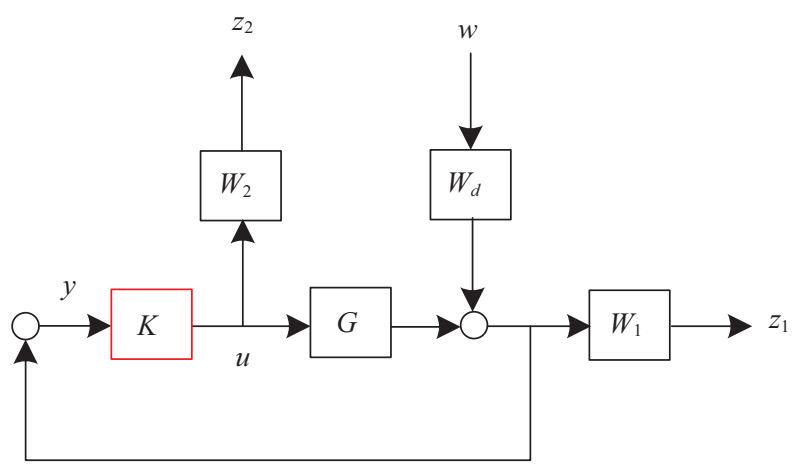

Fig. 3. The mixed sensitivity problem.

$$
\begin{gathered}
\ddot{\theta}=\frac{-m g l M_{e}}{m^{2} l^{2}-\left(m l^{2}+J_{e}\right) M_{e}} \theta+\frac{m l\left(T_{L}+T_{R}\right)}{R m^{2} l^{2}-R M_{e}\left(m l^{2}+J_{e}\right)}, \\
\ddot{x}_{M}=\frac{-\frac{m^{2} l^{2} g}{m l^{2}+J_{e}}}{M_{e}-\frac{m^{2} l^{2}}{m l^{2}+J_{e}}} \theta+\frac{\left(T_{L}+T_{R}\right)}{R\left(M_{e}-\frac{m^{2} l^{2}}{m l^{2}+J_{e}}\right)}, \\
\ddot{\Theta}=\frac{2 R H_{f}\left(T_{L}-T_{R}\right)}{2 R^{2} J_{\Theta}+\left(J_{w}+R^{2} M_{w}\right) H_{f}{ }^{2}},
\end{gathered}
$$

where $M_{e}=M+2 M_{w}+m+\frac{2 J_{w}}{R^{2}}$ and $J_{e}=J_{m \theta}+J_{P \theta}$. Note that the CTWEV is an intrinsic, unstable, and non-minimum phase system.

\section{CONTROLLER DESIGN}

In this section, the proposed CTWEV control scheme will be presented. To carry out $H_{\infty}$ controller synthesis, the chain scattering-matrix description (CSD) approach [12] and [23] will be introduced first.

\section{CSD Approach to $H_{\infty}$ Control}

The CSD theory originated from the conventional electric circuit theory. Due to its benefits of describing a linear system, CSD was gradually extended to the robust control field [12]. Many structures of the CSD have been investigated. The CSD approach proposed in [23] is one of the straightforward and systematic methods utilized to solve the general $H_{\infty}$ control problem based on the coprime factorization framework. According to [23], two types of synthesis approaches can be applied which can obtain the same solutions. Those requesting further information may refer to [23]. In the following controller synthesis procedure, the CSD approach proposed in

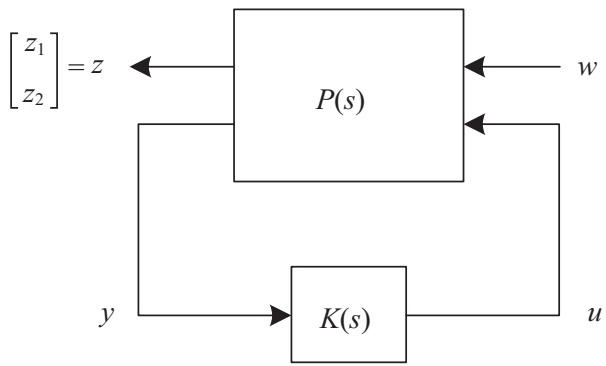

Fig. 4. Standard control configuration.

[23] will be adopted. Now consider a mixed sensitivity problem shown in Fig. 3 as an illustration where $W_{1}, W_{2}$, and $W_{d}$ are weighting functions, respectively. The $G$ plant is $G \stackrel{s}{=}\left[\begin{array}{l|l}A & B \\ \hline C & 0\end{array}\right]$ and the cost function is $\left\|T_{z w}\right\|_{\infty}<\gamma$.

First, formulating this system into the standard control configuration (SCC) as shown in Fig. 4, i.e., the linear fractional transformation (LFT), yields

$$
\operatorname{LFT}(P, K)=P_{11}+P_{12} K\left(I-P_{22} K\right)^{-1} P_{21}
$$

where $P(s)=\left[\begin{array}{c:c}W_{1} W_{d} & W_{1} G \\ 0 & W_{2} \\ \hdashline W_{d} & G\end{array}\right]=\left[\begin{array}{c:c}P_{11} & P_{12} \\ \hdashline P_{21} & P_{22}\end{array}\right]$. Then let $G=\tilde{M}^{-1} \tilde{N}$ be the normalized left coprime factorization of $G$ (i.e., $\tilde{N} \tilde{N}^{\sim}+\tilde{M} \tilde{M}^{\sim}=I$ ), which produces

$$
\begin{aligned}
& {\left[\begin{array}{ll}
\tilde{N} & \tilde{M}
\end{array}\right]^{s}=\left[\begin{array}{c|cc}
A+H C & B & H \\
\hline C & 0 & I
\end{array}\right]} \\
& H=-Y C^{T}
\end{aligned}
$$

where $Y=\operatorname{Ric}\left[\begin{array}{cc}A^{T} & -C^{T} C \\ -B B^{T} & -A\end{array}\right]$ be the solution of the algebraic Riccati equation

$$
A Y+Y A^{T}-Y C^{T} C Y+B B^{T}=0
$$

Here let $W_{d}=\tilde{M}^{-1}$, the system in Fig. 3, be formulated into the state space configuration as Fig. 5.

From Fig. 5, we can determine the augmented system $P(s)$ in SCC as

$$
P(s) \stackrel{s}{=}\left[\begin{array}{c|cc}
A & -H & B \\
\hline\left(\begin{array}{c}
W_{1} C \\
0
\end{array}\right) & \left(\begin{array}{c}
W_{1} \\
0
\end{array}\right) & \left(\begin{array}{c}
0 \\
W_{2}
\end{array}\right) \\
C & I & 0
\end{array}\right]=\left[\begin{array}{c:c}
P_{11} & P_{12} \\
\hdashline P_{21} & P_{22}
\end{array}\right] .
$$




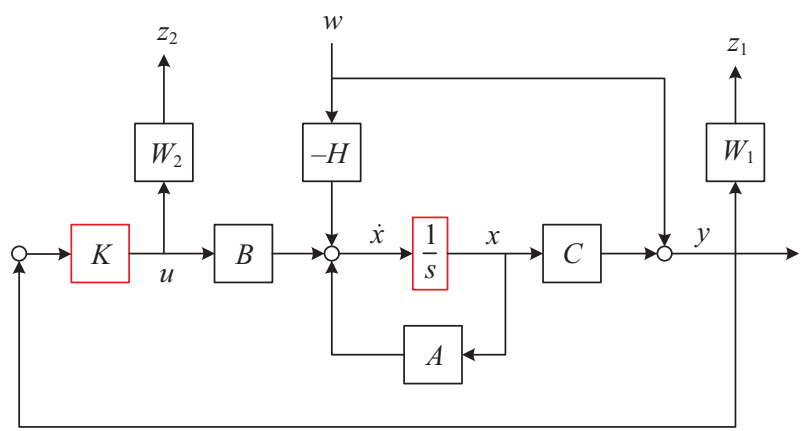

Fig. 5. The state space configuration.

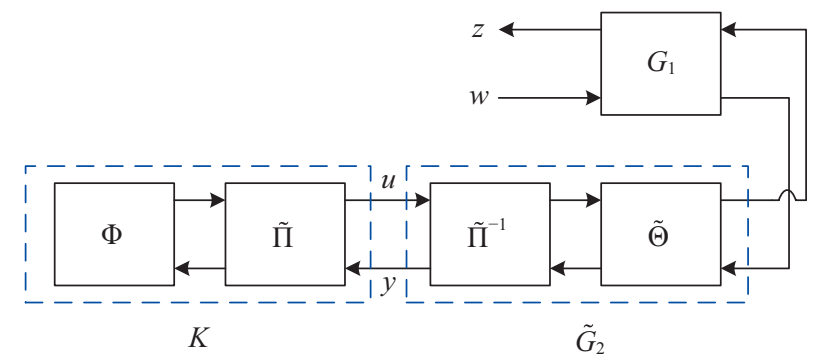

Fig. 6. CSD control configuration.

Note that $P_{21} \stackrel{s}{=}\left[\begin{array}{c|c}A & -H \\ \hline C & I\end{array}\right]=\tilde{M}^{-1}$ is invertible and its inverse is stable. At this stage, by following the synthesis procedure proposed in [23], the LFT can be transformed into the CSD control configuration as shown in Fig. 6 where

$G_{1} \stackrel{s}{=}$

$$
\left[\begin{array}{c|cc}
A+B F_{x} & B_{1} W_{x 12}+B_{2} W_{x 22} & B_{1} W_{x 11}+B_{2} W_{x 21} \\
\hline C_{1}+D_{11} F_{x 1} & D_{11} W_{x 12}+D_{12} W_{x 22} & D_{11} W_{x 11}+D_{12} W_{x 21} \\
+D_{12} F_{x 2} & W_{x 12} & W_{x 11}
\end{array}\right],
$$

$\tilde{G}_{2} \stackrel{s}{=}$

$$
\left[\begin{array}{c|cc}
A+B F_{x} & B_{1} W_{x 12}+B_{2} W_{x 22} & B_{1} W_{x 11}+B_{2} W_{x 21} \\
\hline F_{x 2} & W_{x 22} & W_{x 21} \\
C_{2}+F_{x 1} & W_{x 12} & W_{x 11}
\end{array}\right]=\tilde{\Pi}^{-1} \tilde{\Theta} .
$$

Hence, the closed-loop system from $w$ to $z$ is defined as

$$
\begin{aligned}
z & =\operatorname{CSD}_{r}\left(G_{1}, \operatorname{CSD}_{l}\left(\tilde{G}_{2}, K\right)\right) w \\
& =\operatorname{CSD}_{r}\left(G_{1}, \operatorname{CSD}_{l}\left(\tilde{\Pi}^{-1} \tilde{\Theta}, C S D_{l}(\tilde{\Pi}, \Phi)\right)\right) w
\end{aligned}
$$

where $G_{1}, \tilde{G}_{2}, \tilde{\Theta}$, and $\tilde{\Pi}$ are in $R H_{\infty}$, such that $G_{1}$ is J-lossless and $\tilde{\Theta}$ is dual J-lossless [12]. Solving $G_{1}$ as J-lossless should determine the algebraic Riccati equation to get $F_{x}=\left[\begin{array}{c}F_{x 1} \\ F_{x 2}\end{array}\right]$. Furthermore, $\left[\begin{array}{ll}W_{x 11} & W_{x 12} \\ W_{x 21} & W_{x 22}\end{array}\right]$ is obtained by solving $G_{1}$ as J-lossless. Then applying these conditions into $\tilde{G}_{2}$ can acquire $\tilde{\Theta}=I$ in this problem and

$$
\tilde{\Pi}(s)=\left[\begin{array}{c|cc}
A+H C & -B & H \\
\hline W_{x 22}^{-1} F_{x 2} & W_{x 22}^{-1} & 0 \\
W_{x 11}^{-1}\left(C+F_{x 1}\right) & 0 & W_{x 11}^{-1}
\end{array}\right] .
$$

Therefore the $K(s)$ controller is given by

$$
K(s)=C S D_{l}(\tilde{\Pi}, \Phi), \forall \Phi \in B H_{\infty} .
$$

Thus the central controller (i.e., $\Phi=0$ ) can be obtained as

$$
\begin{aligned}
K_{0}(s) & =C S D_{l}(\tilde{\Pi}, 0)=-\tilde{\Pi}_{11}{ }^{-1} \tilde{\Pi}_{12} \\
& =s\left[\begin{array}{c|c}
A+H C+B F_{x 2} & -H \\
\hline F_{x 2} & 0
\end{array}\right] .
\end{aligned}
$$

\section{Proposed CTWEV Piloting Control System}

Figure 7 shows the proposed CTWEV pilot control scheme where a person is involved in the closed loop to decide the joystick command. The controller of the presented system could be obtained via the aforementioned CSD approach. The following Fig. 8 illustrates the state space configuration of Fig. 7 where the cart's angle of inclination is the only feedback information needed for feedback control.

Let the linearized CTWEV state space model be $\left[\begin{array}{l|l}A & B \\ \hline C & 0\end{array}\right]$, and $H$ is introduced from the normalized coprime factorization. The constants, $W_{1}, W_{2}, W_{3}$, and $W_{4}$ are weighting functions applied to adjust the system performance. It is known that weighting function selection plays an important role in determining a robust controller. Interested readers can refer [21] and therein for advanced discussion about the weighting function selection rules. In Fig. $8, x_{1}=\left[\begin{array}{c}\theta \\ \dot{\theta}\end{array}\right], y_{2}=\theta$, $\bar{u}=\left[\begin{array}{l}T_{L} \\ T_{R}\end{array}\right], \quad A=\left[\begin{array}{ll}0 & 1 \\ a & 0\end{array}\right], \quad B=\left[\begin{array}{ll}0 & 0 \\ b & b\end{array}\right]$, and $C=\left[\begin{array}{ll}1 & 0\end{array}\right]$ where $a=\frac{-m g l M_{e}}{m^{2} l^{2}-\left(m l^{2}+J_{e}\right) M_{e}}, b=\frac{\frac{m l}{R}}{m^{2} l^{2}-M_{e}\left(m l^{2}+J_{e}\right)}$. As mentioned in the introduction, this CTWEV control scheme 


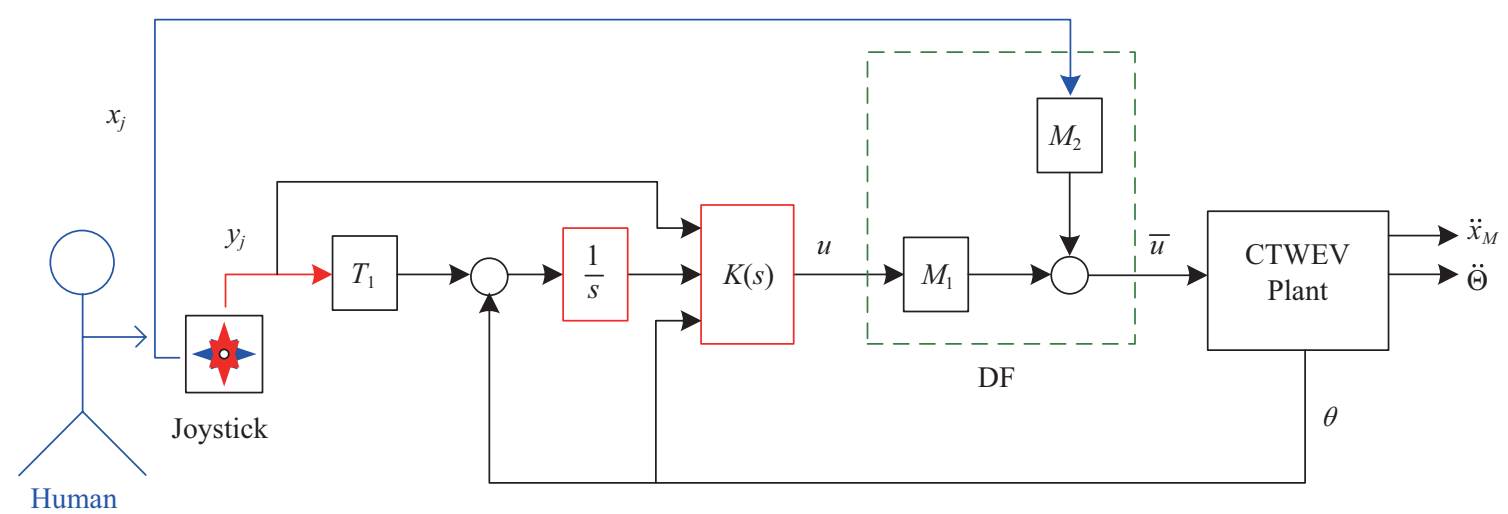

Fig. 7. The proposed CTWEV pilot control system.

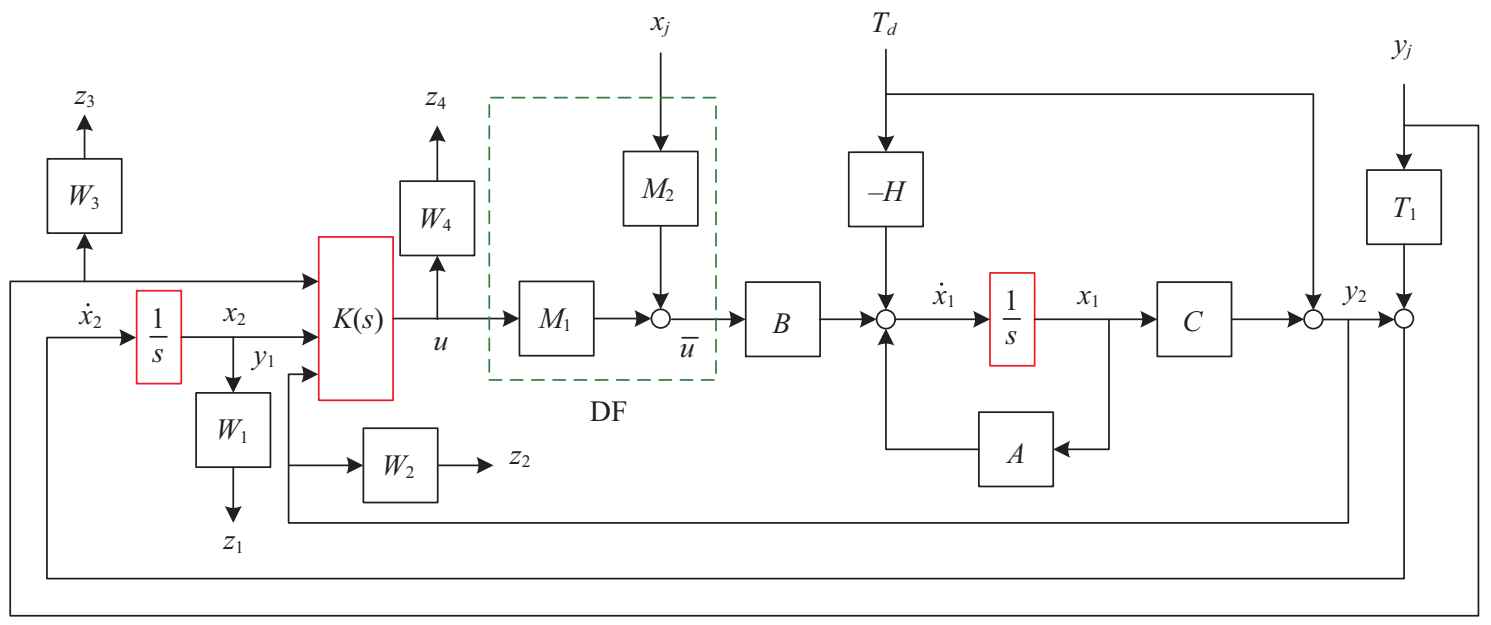

Fig. 8. State space configuration of proposed CTWEV system.

could be remote-controlled when linked with a joystick. The $y_{j}$ input is the acceleration command of $x_{M}, x_{j}$ denotes the acceleration command of $\Theta$, and $T_{d}$ represents the disturbance. Here from Fig. 8, we have the augmented system $P(s)$ as

$$
\left[\begin{array}{l}
\dot{x}_{1} \\
\dot{x}_{2} \\
z_{1} \\
z_{2} \\
z_{3} \\
z_{4} \\
y_{1} \\
y_{2} \\
y_{j}
\end{array}\right]=\left[\begin{array}{cccccc}
A & 0 & -H & 0 & B M_{2} & B M_{1} \\
C & 0 & I & T_{1} & 0 & 0 \\
0 & W_{1} & 0 & 0 & 0 & 0 \\
W_{2} C & 0 & W_{2} & 0 & 0 & 0 \\
0 & 0 & 0 & W_{3} & 0 & 0 \\
0 & 0 & 0 & 0 & 0 & W_{4} \\
0 & I & 0 & 0 & 0 & 0 \\
C & 0 & I & 0 & 0 & 0 \\
0 & 0 & 0 & I & 0 & 0
\end{array}\right]\left[\begin{array}{c}
x_{1} \\
x_{2} \\
T_{d} \\
y_{j} \\
x_{j} \\
u
\end{array}\right]
$$

where $w=\left[\begin{array}{lll}T_{d} & y_{j} & x_{j}\end{array}\right]^{T}$ and $z=\left[\begin{array}{llll}z_{1} & z_{2} & z_{3} & z_{4}\end{array}\right]^{T}$.

After acquiring the $P(s)$ augmented system, the CSD approach presented in the previous subsection can be used.
Consequently, applying $P(s)$ into the CSD synthesis procedure, solving the $H_{\infty}$ control problem (i.e., $\left\|T_{z w}\right\|_{\infty}<\gamma$ ), and obtaining a robust $K(s)$ controller become feasible as seen in (15).

Note that Fig. 8 involves an electronic differential steering rule DF (i.e., the dashed box in Fig. 8) which can distribute the torque command to both the left and right wheel motors. The CTWEV is driven by two individual actuators; thus the electronic differential algorithm becomes indispensable. Some electronic differential algorithms developed for two-wheeled mobile robots can also be found in $[2,6,7,8,18,22]$. The torque commands $\left(T_{L}, T_{R}\right)$ from inputs $\left(u, x_{j}\right)$ to the left and right driving motors, respectively, are given by

$$
\left[\begin{array}{l}
T_{L} \\
T_{R}
\end{array}\right]=\left[\begin{array}{ll}
M_{1} & M_{2}
\end{array}\right]\left[\begin{array}{l}
u \\
x_{j}
\end{array}\right] .
$$

This differential steering algorithm for motion and orientation control involves matrix $\left[M_{1} M_{2}\right]$ which is introduced to allow for the association between two different physical quantities and to make the pilot control more flexible. Note that, the matrix $M_{2}$ is adjustable by human, which enhances 


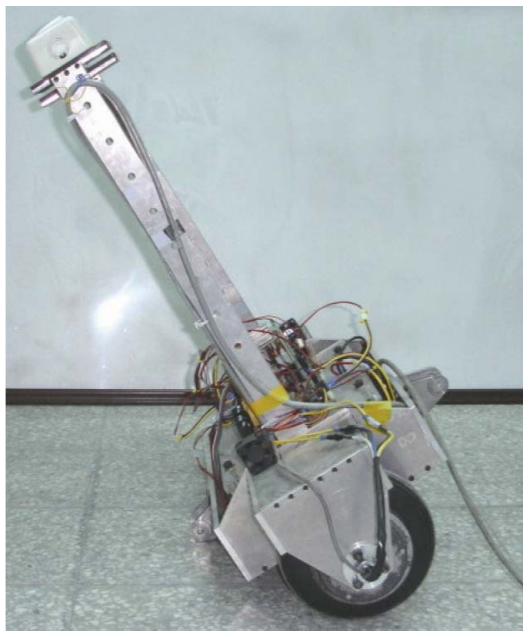

Fig. 9. Rest posture of CTWEV.

the motion sensitivity of the controlled CTWEV. In addition, the $T_{1}$ constant in Fig. 8 is set up to adjust the cart motion forward and backward according to different ground friction conditions.

\section{Rest Posture and Auto-standup of CTWEV}

Actually, the linear $K(s)$ controller was designed to operate the CTWEV within the equilibrium point. Besides, the CTWEV is controlled remotely. How the auto-balancing robots support themselves are currently prevalent issues such as [1] and [15]. Similar "swing up" issues appear in the conventional inverted pendulum. Figure 9 shows the "rest" posture of the proposed CTWEV. This is the primary position of the cart before operation. Moreover, in order to save battery power in the standby mode for transportation applications, the CTWEV will assume a rest posture where the CTWEV will achieve a large inclined angle. The maximum angle of inclination can be designed according to the relative height of the cart chassis. For example, the rest angle of inclination of the proposed CTWEV on the level ground is $30^{\circ}$.

In the proposed system, the CTWEV is allowed to be in the rest posture. In addition, it should be able to right itself. There are many kinds of strategies that can initiate standing commands in auto-balancing robots. If the rest angle of inclination is not so acute, the $K(s)$ controller is qualified to prop the CTWEV. In the following example, we will discuss the limitation of using $K(s)$ to execute upright placement.

\section{EXAMPLE AND DISCUSSION}

In the following example, the model parameters of Fig. 2 for the CTWEV were obtained from [22], which are given by $J_{m \theta}=0.153\left(\mathrm{Kgm}^{2}\right), J_{P \theta}=0.125\left(\mathrm{Kgm}^{2}\right), M_{w}=5.44(\mathrm{Kg}), M=$ $15.747(\mathrm{Kg}), J_{w}=0.013\left(\mathrm{Kgm}^{2}\right), m=4(\mathrm{Kg}), l=0.53(m), J_{\Theta}=$ $0.576\left(\mathrm{Kgm}^{2}\right), H_{f}=0.44(m)$, and $R=0.1(m)$. Hence the state space model parameters in Fig. 7 are

$$
\begin{aligned}
& {\left[\begin{array}{l|l}
A & B \\
\hline C & D
\end{array}\right]=\left[\begin{array}{cc|cc}
0 & 1 & 0 & 0 \\
16.406 & 0 & -0.504 & -0.504 \\
\hline 1 & 0 & 0 & 0
\end{array}\right],} \\
& H=\left[\begin{array}{c}
-8.103 \\
-32.828
\end{array}\right] .
\end{aligned}
$$

The DF matrix in this study is set as

$$
\left[\begin{array}{ll}
M_{1} & M_{2}
\end{array}\right]=\left[\left(\begin{array}{l}
0.5 \\
0.5
\end{array}\right)\left(\begin{array}{c}
0.1396 \\
-0.1396
\end{array}\right)\right]
$$

Letting $W_{1}=0.08, W_{2}=0.1, W_{3}=1, W_{4}=0.0035$, and $T_{1}=$ -0.0923 . Then the $P(s)$ augmented system were determined. Given $\gamma=1.01$ and applying $P(s)$ into the aforementioned CSD synthesis procedure could obtain a central $K(s) \stackrel{s}{=}\left[\begin{array}{l|l}A_{k} & B_{k} \\ \hline C_{k} & D_{k}\end{array}\right]$ controller as

$$
\begin{aligned}
A_{k} & =\left[\begin{array}{rrr}
1.6605 & 35.9107 & 50.0370 \\
-2.2526 & -10.0517 & -12.0097 \\
-2.8091 & -10.7648 & -12.4188
\end{array}\right], \\
B_{k} & =\left[\begin{array}{rrr}
-4.1626 \mathrm{e}-008 & 3.8217 & -0.0004 \\
-1.9436 \mathrm{e}-006 & -1.3404 & -0.0089 \\
2.2828 \mathrm{e}-006 & -1.7356 & 0.0106
\end{array}\right], \\
C_{k} & =\left[\begin{array}{lll}
16.6037 & -433.9971 & -684.8680
\end{array}\right], \\
D_{k} & =\left[\begin{array}{lll}
0 & 0 & 0
\end{array}\right] .
\end{aligned}
$$

The following simulation assumes that the ground friction is compensated for, and its evaluations are based on the nonlinear CTWEV model (1)-(3). The joystick commands are set as $\left(x_{j}, y_{j}\right)=(1 * \sin (0.1 * 2 \pi), 1)$ and $T_{d}=0$. Figure 10(a) shows the system response of the proposed CTWEV system. The simulation result reveals an acceptable performance. Note that as clearly seen in Fig. 10(a), the undershooting phenomenon of an inherent non-minimum phase system exists. Let $\left(x_{j}, y_{j}\right)=(0,0)$, Fig. 10(b) illustrates the performance with respect to the step disturbance added in the pendulum part of the CTWEV. As can be seen in Fig. 10(b), after the release of disturbance torque, the CTWEV's angle of inclination will return to its balance angle.

The following simulation evaluates the limitations of the CTWEV auto-standup ability. If the practical saturation condition is omitted, Fig. 11(a) shows the auto-standup behavior of the proposed system from the rest posture on the level ground. As seen in this figure, the CTWEV can right itself 

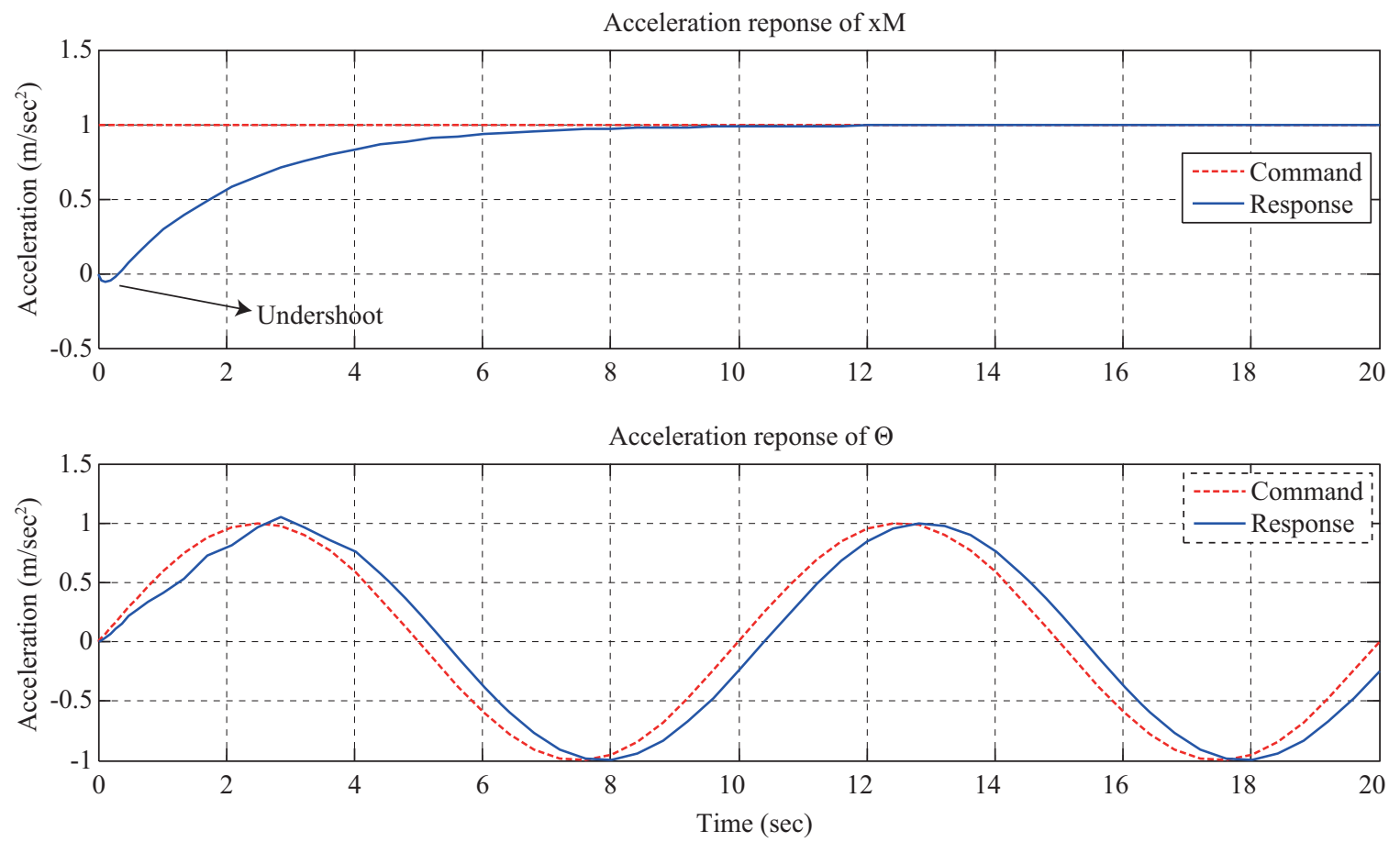

(a) Performance test.
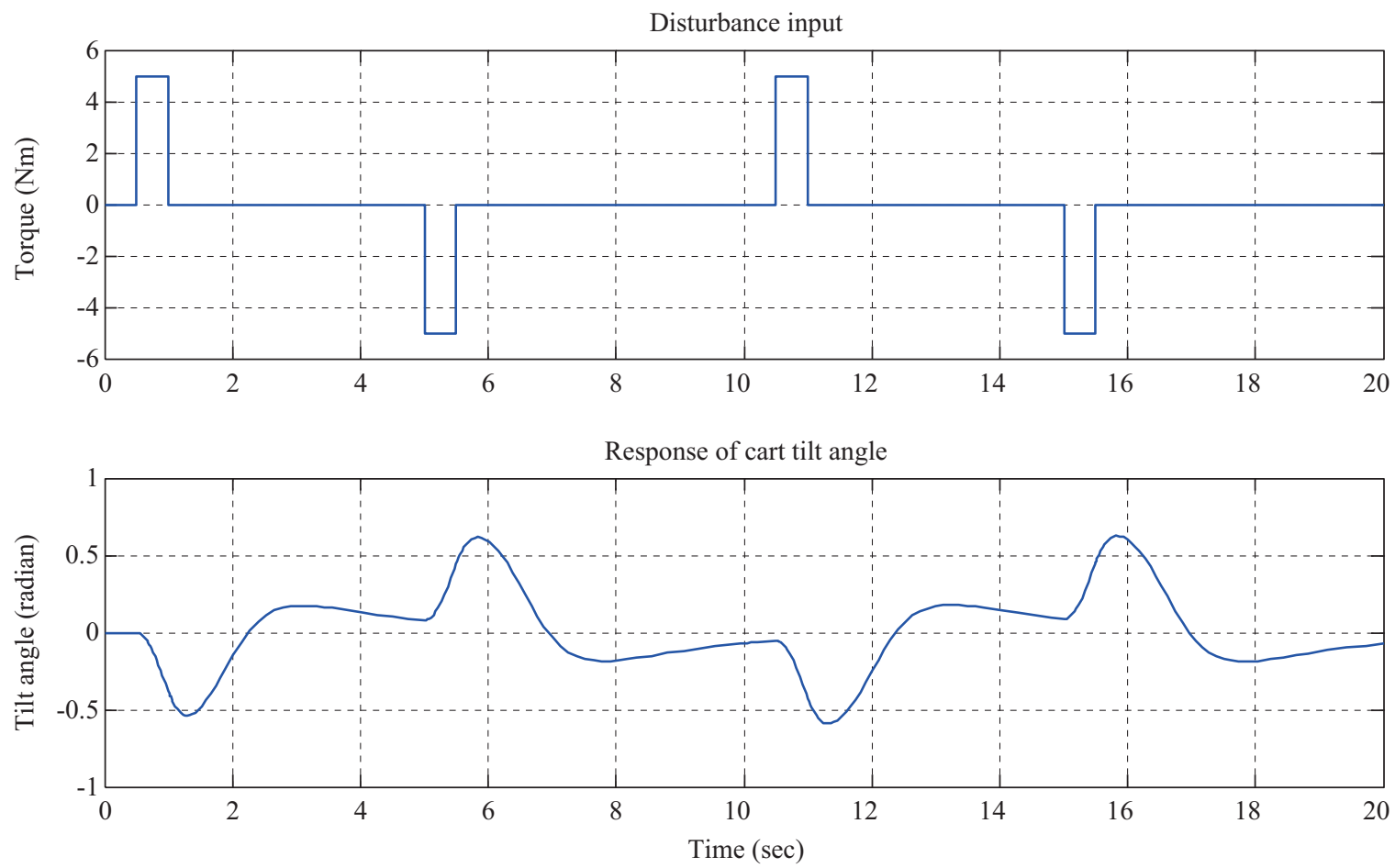

(b) Disturbance rejection test.

Fig. 10. Simulations.

using the $K(s)$ controller. Figure 11(b) shows the existing limit cycle when the disturbance torque causes an inclined displacement from the equilibrium point larger than 1 radian (i.e., about $57.3^{\circ}$ ). This is also the maximum acute angle of incli- nation that the proposed CTWEV can encounter and still be able to correct for and right itself. Note that if the proposed CTWEV tilts sharper than 1 radian, the cart will continue oscillating and will not reach the equilibrium point even when 


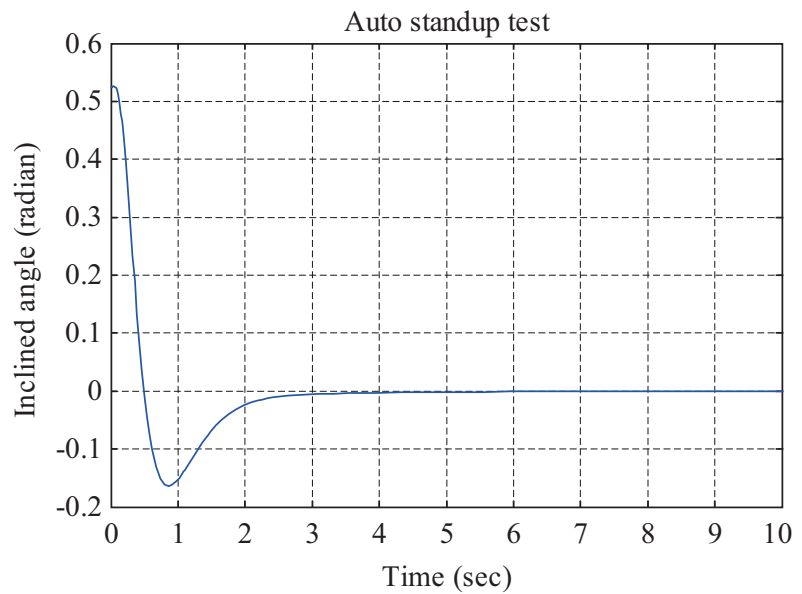

(a) Auto-standup test.

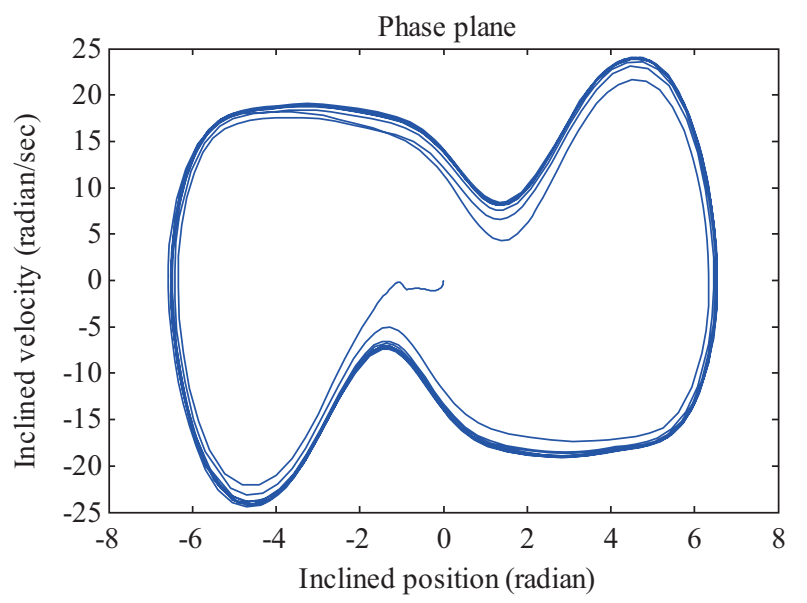

(b) Limit cycle.

Fig. 11. Performance test

the disturbance has been removed. The simulation results of Fig. 10 also mean that: using such a type of feedback control setup, the limitation is the angle of inclination and cannot be larger than 1 radian. This is the primary limitation when stabilizing an inverted pendulum type mobile robot solely based on the cart's angle of inclination.

From the simulation results in Figs. 10 and 11, we know that the proposed CTWEV control scheme is qualified to perform control tasks and it can auto-standup from the rest posture. In addition, the actuator saturation problem, integration windup, and nonlinear disturbances such as ground friction and mechanism backlash will affect system performance significantly and all should be compensated for in practical implementations. Figure 12 shows the video clips of experimental results of auto-standup and cost-effective CTWEV. In Fig. 12(a), it is obvious that the proposed system can autostandup in a very short time. In Fig. 12(b), the proposed scheme proved that the CTWEV can be piloted solely based on cart's angle of inclination. These demonstrations are also available for viewing on the website [3] and have confirmed that the proposed approach is valid.

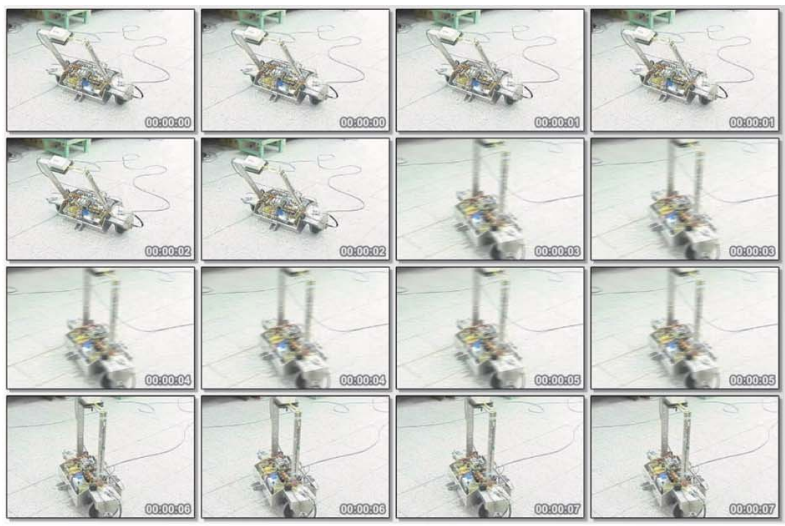

(a) Auto-standup test.

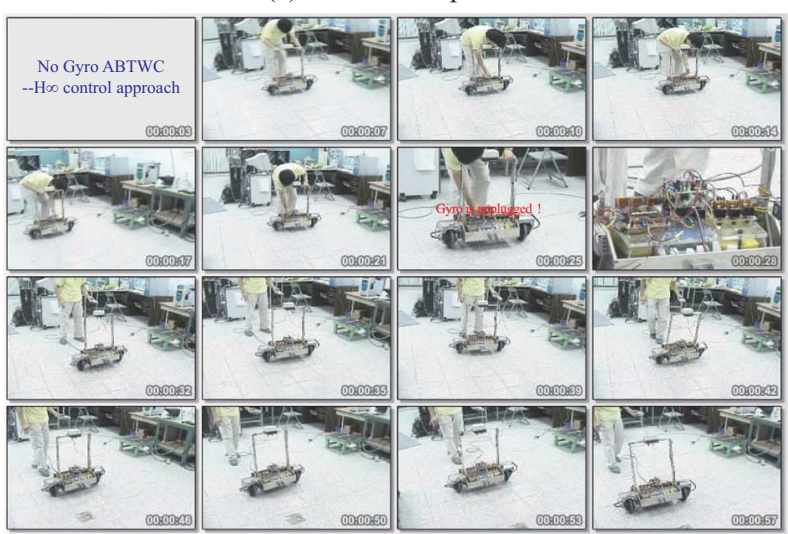

(b) Pilot control test.

Fig. 12. Experimental video clips.

\section{CONCLUSIONS}

The CTWEV is an inherently unstable, large-scale, MIMO, and non-minimum phase system. This paper has presented a robust control scheme that can pilot the CTWEV via a remote solely based on cart's angle of inclination. The proposed scheme embedded an electronic differential steering algorithm, which was developed for coordinating cart motion. The chain scattering description approach to the $H_{\infty}$ control problem has also been presented. Finally, through the simulation results and practical demonstration video clips, the illustrated system has shown its feasibility and flexibility for CTWEV remote pilot motion.

\section{ACKNOWLEDGMENTS}

This work was supported by the National Science Council (NSC) of Taiwan, R.O.C., under grant project NSC 93-2213E-006-008. The authors would like to thank Mr. Michael J. Newkirk and anonymous reviewers for helping to improve the quality of this paper.

\section{REFERENCES}

1. Au, S. K. W., Xu, Y., and Yu, W. W. K., "Control of tilt-up motion of a 
single wheel robot via model-based and human-based controllers," Mechatronics, Vol. 11, pp. 451-473 (2001).

2. Building a Balancing Scooter, http://tlb.org/scooter.html.

3. Control of an Auto-balancing Two-wheeled Cart, http://www.me.ncku. edu.tw/ abtwc/.

4. Doyle, J. C., Glover, K., Khargonekar, P. P., and Francis, B. A., "Statespace solutions to standard $H_{2}$ and $H_{\infty}$ control problems," IEEE Transactions on Automatic Control, Vol. 34, pp. 831-847 (1989).

5. Feng, Q. and Yamafuji, K., "Design and simulation of control systems of an inverted pendulum," Robotica, Vol. 6, pp. 235-241 (1988).

6. Grasser, F., D'Arrigo, A., Colombi, S., and Ruffer, A. C., "JOE: a mobile, inverted pendulum," IEEE Transactions on Industrial Electronics, Vol. 49, pp. 107-114 (2002).

7. Ha, Y.-S. and Yuta, S., "Trajectory tracking control for navigation of the inverse pendulum type self-contained mobile robot," Robotics and Autonomous Systems, Vol. 17, pp. 65-80 (1996).

8. Hu, J.-S. and Tsai, M.-C., Control and Fault Diagnosis of an Autobalancing Two-wheeled Cart: Remote Pilot and Sensor/Actuator Fault Diagnosis for Coaxial Two-wheeled Electric Vehicle, Saarbrücken: VDM Verlag (2008).

9. Human Transporter, http://www.segway.com/.

10. iBOT Mobility System, http://www.independence now.com/ibot/.

11. Karkoub, M. A. and Parent, M., "Modeling and non-linear feedback stabilization of a two-wheel vehicle," Proceedings of the Institution of Mechanical Engineers, Part I, Systems and Control Engineering, Vol. 218, pp. 675-686 (2004).

12. Kimura, H., Chain-Scattering Approach to $H_{\infty}$ Control, Boston: Birkhäuser (1996).

13. Ko, A., Lau, H. Y. K., and Lau, T. L., "SOHO security with mini selfbalancing robots," Industrial Robot, Vol. 32, pp. $492-498$ (2005).

14. Luo, R. C. and Chen, T.-M., "Development of a multi-behavior based mobile robot for remote supervisory control through the internet," IEEE/ASME Transactions on Mechatronics, Vol. 5, pp. 376-385 (2000).

15. Morimoto, J. and Doya, K., "Reinforcement learning of dynamic motor sequence: learning to standup," Proceedings of IEEE/RSJ International Conference on Intelligent Robots and Systems, Victoria, pp. 1721-1726 (1998).

16. nBot Balancing Robot, http://www.geology.smu.edu/ dpa-www/robo/ nbot/.

17. Pathak, K., Franch, J., and Agrawal, S. K., "Velocity and position control of a wheeled inverted pendulum by partial feedback linearization," IEEE Transactions on Robotics, Vol. 21, pp. 505-513 (2005).

18. Ren, T.-J., Chen, T.-C., Tsai, M.-C., and Yao, W.-S., "Modeling and motion control of the mobile vehicle with an inverted pendulum," Proceedings of International Conference on Intelligent Manipulation and Grasping, Genoa, pp. 455-460 (2004).

19. Shiroma, N., Matsumoto, O., and Tani, K., "Cooperative behavior of a mechanically unstable mobile robot for object transportation," JSME International Journal, Series C, Vol. 42, pp. 965-973 (1999).

20. The Japan Times, http://www.japantimes.co.jp/cgi-bin/getarticle.p15?Nn 20011231a9.htm.

21. Tsai, M.-C., Geddes, E. J. M., and Postlethwaite, I., "Pole-zero cancellations and closed-loop properties of an $H_{\infty}$ mixed sensitivity design problem," Automatica, Vol. 28, pp. 519-530, 1992.

22. Tsai, M.-C. and Hu, J.-S., "Pilot control of an auto-balancing twowheeled cart," Advanced Robotics, Vol. 21, pp. 817-827 (2007).

23. Tsai, M.-C. and Tsai, C.-S., "A chain scattering matrix description approach to $H_{\infty}$ control," IEEE Transactions on Automatic Control, Vol. 38, pp. 1416-1421 (1993).

24. Urakubo, T., Tsuchiya, K., and Tsujita, K., "Motion control of a two-wheeled mobile robot," Advanced Robotics, Vol. 15, pp. 711-728 (2001). 